Proyecciones Journal of Mathematics

Vol. 35, No 2, pp. 225-233, June 2016.

Universidad Católica del Norte

Antofagasta - Chile

\title{
Approximate Drygas mappings on a set of measure zero
}

\author{
Muaadh Almahalebi \\ Ibn Tofail University, Morocco \\ Received : October 2015. Accepted: April 2016
}

\begin{abstract}
Let $\mathbf{R}$ be the set of real numbers, $Y$ be a Banach space and $f$ : $\mathbf{R} \rightarrow Y$. We prove the Hyers-Ulam stability for the Drygas functional equation

$$
f(x+y)+f(x-y)=2 f(x)+f(y)+f(-y)
$$

for all $(x, y) \in \Omega$, where $\Omega \subset \mathbf{R}^{2}$ is of Lebesgue measure 0 .

Keywords: Drygas functional equation; stability; Baire category theorem; First category; Lebesgue measure.
\end{abstract}

2000 Mathematics Subject Classification: 39B82. 


\section{Introduction}

To obtain a Jordan and von Neumann type characterization theorem for the quasi-inner-product spaces, Drygas [12] considered the functional equation

$$
f(x+y)+f(x-y)=2 f(x)+f(y)+f(-y)
$$

for all $x, y \in \mathbf{R}$. However, the general solution of this functional equation was given by Ebanks, Kannappan and Sahoo [13] as

$$
f(x)=A(x)+Q(x),
$$

where $A: \mathbf{R} \longrightarrow \mathbf{R}$ is an additive function and $Q: \mathbf{R} \longrightarrow \mathbf{R}$ is a quadratic function.

In 2002, S. M. Jung and P. K. Sahoo [18] considered the stability problem of the following functional equation:

$$
f(x+y)+f(x-y)=2 f(x)+f(y)+g(2 y),
$$

and as a consequence they obtained the stability theorem of functional equation of Drygas (1.1) where $f$ and $g$ are functions from a real vector space $X$ to a Banach space $Y$.

Here we state a slightly modified version of the results in [18].

Theorem 1.1. Let $\varepsilon \geq 0$ be fixed and let $X$ be a real vector space and $Y$ a Banach space. If a function $f: X \longrightarrow Y$ satisfies the inequality

$$
\|f(x+y)+f(x-y)-2 f(x)-f(y)-f(-y)\| \leq \varepsilon,
$$

for all $x, y \in X$, then there exists a unique additive mapping $A: X \longrightarrow Y$ and a unique quadratic mapping $Q: X \longrightarrow Y$ such that $S=A+Q$ is a solution of (1.1) such that

$$
\|f(x)-S(x)\| \leq \frac{25}{3} \varepsilon \text { for all } x \in X .
$$

This result was improved first by Yang in [27] and later by Sikorska in [26]. In this paper we use the Sikorska's result as a basic tool in the main result. So, we need to present the following theorem.

Theorem 1.2. [26] Let $(X,+)$ be a group and $Y$ be a Banach space. Given an $\varepsilon>0$, assume that $f: X \rightarrow Y$ satisfies the condition

$$
\|f(x+y)+f(x-y)-2 f(x)-f(y)-f(-y)\| \leq \varepsilon, \quad x, y \in X .
$$


Then there exists a uniquely determined function $g: X \rightarrow Y$ such that

$$
g(x)=\frac{2}{9} g(3 x)-\frac{1}{9} g(-3 x), \quad x \in X
$$

and

$$
\|f(x)-g(x)\| \leq \varepsilon \quad x \in X .
$$

Moreover, if $X$ is Abelian, then $g$ satisfies

$$
g(x+y)+g(x-y)=2 g(x)+g(y)+g(-y), \quad x, y \in X .
$$

The stability and solution of the Drygas equation under some additional conditions was also studied by Forti and Sikorska in [15] in the case when $X$ and $Y$ are amenable groups.

It is a very natural subject to consider functional equations or inequalities satisfied on restricted domains or satisfied under restricted conditions [1]-[8], [11], [14]-[17], [19], [20], [23]-[25]. Among the results, S. M. Jung and J. M. Rassias proved the Hyers-Ulam stability of the quadratic functional equations in a restricted domain [17], [22].

It is very natural to ask if the restricted domain $D:=\left\{(x, y) \in X^{2}\right.$ : $\|x\|+\|y\| \geq d\}$ can be replaced by a much smaller subset $\Omega \subset D$, i.e., a subset of measure 0 in a measure space $X$. In 2013, J. Chung considered the stability of the Cauchy functional equation

$$
f(x+y)=f(x)+f(y)
$$

in a set $\Omega \subset\left\{(x, y) \in \mathbf{R}^{2}:|x|+|y| \geq d\right\}$ of measure $m(\Omega)=0$ when $f: \mathbf{R} \longrightarrow \mathbf{R}$. In 2014, J. Chung and J. M. Rassias proved the stability of the quadratic functional equation in a set of measure zero.

In this paper, we prove the Hyers-Ulam stability theorem for the Drygas functional equation (1.1) in $\Omega \subset X^{2}$ of Lebesgue measure 0 .

\section{General approach}

Through this paper, we denote by $X$ and $Y$ a real normed space and a real Banach space. For given $x, y, a \in X$, we define

$$
P_{x, y, a}:=\{(x+y, a),(x-y, a),(x, y+a),(x, y-a),(y, a),(-y,-a)\}
$$

Let $\Omega \subset X^{2}$. Throughout this section, we assume that $\Omega$ satisfies the condition: For given $x, y \in X$, there exists $a \in X$ such that 


$$
P_{x, y, a} \subset \Omega \text {. }
$$

In the following, we prove the Hyers-Ulam stability theorem for the Drygas functional equation (1.1) in $\Omega$.

Theorem 2.1. Let $\varepsilon \geq 0$ be fixed. Suppose that $f: X \longrightarrow Y$ satisfies the functional inequality

$$
\|f(x+y)+f(x-y)-2 f(x)-f(y)-f(-y)\| \leq \varepsilon
$$

for all $(x, y) \in \Omega$. Then there exists a unique mapping $g: X \longrightarrow Y$ such that $g$ is a solution of (1.1) and

$$
\|f(x)-g(x)\| \leq 3 \varepsilon
$$

for all $x \in X$.

Proof. Let $D(x, y)=f(x+y)+f(x-y)-2 f(x)-f(y)-f(-y)$. Since $\Omega$ satisfies $(C)$, for given $x, y \in X$, there exists $a \in X$ such that

$$
\begin{array}{lll}
\|D(x+y, a)\| \leq \varepsilon, & \|D(x-y, a)\| \leq \varepsilon, & \|D(x, y+a)\| \leq \varepsilon, \\
\|D(x, y-a)\| \leq \varepsilon, & \|D(y, a)\| \leq \varepsilon, & \|D(-y,-a)\| \leq \varepsilon .
\end{array}
$$

Thus, using the triangle inequality we have

$$
\begin{gathered}
\|f(x+y)+f(x-y)-2 f(x)-f(y)-f(-y)\|=\|-\frac{1}{2} D(x+y, a)-\frac{1}{2} D(x-y, a) \\
+\frac{1}{2} D(x, y+a)+\frac{1}{2} D(x, y-a)+\frac{1}{2} D(y, a)+\frac{1}{2} D(-y,-a) \| \leq 3 \varepsilon
\end{gathered}
$$

for all $x, y \in X$. Next, according Theorem 1.1, there exists a unique mapping $g: X \longrightarrow Y$ such that

$$
\|f(x)-g(x)\| \leq 3 \varepsilon
$$

for all $x \in X$. This completes the proof. $\square$ The following corollary is a particular case of Theorem 2.1, where $\varepsilon=0$.

Corollary 2.2. Suppose that $f: X \longrightarrow Y$ satisfies the functional equation

$$
f(x+y)+f(x-y)=2 f(x)+f(y)+f(-y)
$$

for all $(x, y) \in \Omega$. Then, (2.3) holds for all $x, y \in X$. 


\section{Construction of a set $\Omega$ of Lebesgue measure zero}

In this section we construct a set $\Omega$ of measure zero satisfying the condition $(C)$ when $X=\mathbf{R}$. From now on,we identify $\mathbf{R}^{2}$ with $\mathbf{C}$. The following lemma is a crucial key of our construction [[22], Theorem 1.6].

Lemma 3.1. The set $\mathbf{R}$ of real numbers can be partitioned as $\mathbf{R}=F \cup K$ where $F$ is of first Baire category, i.e., $F$ is a countable union of nowhere dense subsets of $\mathbf{R}$, and $K$ is of Lebesgue measure 0 .

The following lemma was proved by J. Chung and J. M. Rassias in [9] and [10].

Lemma 3.2. Let $K$ be a subset of $\mathbf{R}$ of measure 0 such that $K^{c}:=\mathbf{R} \backslash K$ is of first Baire category. Then, for any countable subsets $U \subset \mathbf{R}, V \subset \mathbf{R} \backslash\{0\}$ and $M>0$, there exists $a \geq M$ such that

$$
U+a V=\{u+a v: u \in U, v \in V\} \subset K .
$$

In the following theorem, we give the construction of a set $\Omega$ of Lebesgue measure zero.

Theorem 3.3. Let $\Omega=e^{-\frac{\pi}{6} i}(K \times K)$ be the rotation of $K \times K$ by $-\frac{\pi}{6}$, i.e.,

$$
\Omega=\left\{(p, q) \in \mathbf{R}^{2}: \frac{\sqrt{3}}{2} p-\frac{1}{2} q \in K, \frac{1}{2} p+\frac{\sqrt{3}}{2} q \in K\right\} .
$$

Then $\Omega$ satisfies the condition $(C)$ which has two-dimensional Lebesgue measure 0 .

Proof. By the construction of $\Omega$, the condition $(C)$ is equivalent to the condition that for every $x, y \in \mathbf{R}$, there exists $a \in \mathbf{R}$ such that

$$
e^{-\frac{\pi}{6} i} P_{x, y, a} \subset K \times K \text {. }
$$

The inclusion (3.3) is equivalent to

$$
S_{x, y, a}:=\left\{\frac{\sqrt{3}}{2} u-\frac{1}{2} v, \frac{1}{2} u+\frac{\sqrt{3}}{2} v:(u, v) \in P_{x, y, a}\right\} \subset K .
$$

It is easy to check that the set $S_{x, y, a}$ is contained in a set of form $U+a V$, where

$U=\left\{\frac{\sqrt{3}}{2}(x+y), \frac{\sqrt{3}}{2}(x-y), \frac{1}{2}(x+y), \frac{1}{2}(x-y),\left(\frac{\sqrt{3}}{2} x-\frac{1}{2} y\right),\left(\frac{1}{2} x+\frac{\sqrt{3}}{2} y\right)\right.$, 


$$
\begin{gathered}
\left.\frac{\sqrt{3}}{2} y,-\frac{\sqrt{3}}{2} y, \frac{1}{2} y,-\frac{1}{2} y\right\}, \\
V=\left\{\frac{1}{2},-\frac{1}{2}, \frac{\sqrt{3}}{2},-\frac{\sqrt{3}}{2}\right\} .
\end{gathered}
$$

By Lemma 3.2, for given $x, y \in \mathbf{R}$ and $M>0$ there exists $a \geq M$ such that

$$
S_{x, y, a} \subset U+a V \subset K .
$$

Thus, $\Omega$ satisfies $(C)$. This completes the proof.

Corollary 3.4. Suppose that $f: \mathbf{R} \longrightarrow \mathbf{R}$ satisfies

$$
|f(x+y)+f(x-y)-2 f(x)-f(y)-f(-y)| \longrightarrow 0
$$

as $(x, y) \in \Omega,|x|+|y| \longrightarrow \infty$. Then $f$ is a Drygas mapping.

Proof. The condition (3.6) implies that for each $n \in \mathbf{N}$, there exists $d_{n}>0$ such that

$$
|f(x+y)+f(x-y)-2 f(x)-f(y)-f(-y)| \leq \frac{1}{n}
$$

for all $(x, y) \in \Omega_{d_{n}}:=\left\{(x, y) \in \Omega:|x|+|y| \geq d_{n}\right\}$. In view of the proof of Theorem 2.1, the inclusion (3.5) implies that for every $x, y \in \mathbf{R}$ and $M>0$ there exists $a \geq M$ such that

$$
P_{x, y, a} \subset \Omega \text {. }
$$

For given $x, y \in \mathbf{R}$ if we take $M=d_{n}+|x|+|y|$ and if $a \geq M$, then we have

$$
P_{x, y, a} \subset\left\{(p, q):|x|+|y| \geq d_{n}\right\} .
$$

It follows from (3.8) and (3.9) that for every $x, y \in \mathbf{R}$ there exists $a \in \mathbf{R}$ such that

$$
P_{x, y, a} \subset \Omega_{d_{n}} .
$$

So, $\Omega_{d_{n}}$ satisfies the condition $(C)$. Thus, by Theorem 2.1, there exists a unique additive mapping $A: \mathbf{R} \longrightarrow \mathbf{R}$ and a unique quadratic mapping $Q: \mathbf{R} \longrightarrow \mathbf{R}$ such that

$$
\left\|f(x)-A_{n}(x)-Q_{n}(x)\right\| \leq \frac{25}{n}
$$


for all $x \in \mathbf{R}$. Replacing $n \in \mathbf{N}$ by $m \in \mathbf{N}$ in (3.11) and using the triangle inequality we have

$$
-\mathrm{A}_{n}(x)-A_{m}(x)+Q_{n}(x)-Q_{m}(x)|\leq| A_{n}(x)+Q_{n}(x)-f(x)|+| f(x)-
$$
$A_{m}(x)-Q_{m}(x)$

$\leq \frac{25}{n}+\frac{25}{m} \leq 50$

for all $m, n \in \mathbf{N}$ and $x \in \mathbf{R}$. Hence, $A_{n}+Q_{n}-A_{m}-Q_{m}$ is bounded. So, we get that

$$
A_{n}+Q_{n}(x)=A_{m}+Q_{m}(x)
$$

for all $m, n \in \mathbf{N}$. Then, $A_{n}=A_{m}$ and $Q_{n}=Q_{m}$ for all $m, n \in \mathbf{N}$. Now, letting $n \longrightarrow \infty$ in (3.11) we get the result.

\section{References}

[1] C. Alsina, J. L. Garcia-Roig, On a conditional Cauchy equation on rhombuses, in: J.M. Rassias (Ed.), Functional Analysis, Approximation Theory and Numerical Analysis, World Scientific, (1994).

[2] A. Bahyrycz, J. Brzdȩk, On solutions of the d'Alembert equation on a restricted domain, Aequationes Math. 85, pp. 169-183, (2013).

[3] B. Batko, Stability of an alternative functional equation, J. Math. Anal. Appl. 339, pp. 303-311, (2008).

[4] B.Batko, On approximation of approximate solutions of Dhombres equation, J. Math. Anal. Appl. 340, pp. 424-432, (2008).

[5] J. Brzdęk, On the quotient stability of a family of functional equations, Nonlinear Anal. 71, pp. 4396-4404, (2009).

[6] J. Brzdęk, On a method of proving the Hyers-Ulam stability of functional equations on restricted domains, Aust. J. Math. Anal. Appl. 6, pp. 1-10, (2009).

[7] J. Brzdęk, J. Sikorska, A conditional exponential functional equation and its stability, Nonlinear Anal. 72, 2929-2934, (2010).

[8] J. Chung, Stability of functional equations on restricted domains in a group and their asymptotic behaviors, Comput. Math. Appl. 60, pp. 2653-2665, (2010). 
[9] J. Chung, Stability of a conditional Cauchy equation on a set of measure zero, Aequationes Math. (2013), http://dx.doi.org/ $10.1007 / \mathrm{s} 00010-013-0235-5$.

[10] J. Chung and J. M. Rassias, Quadratic functional equations in a set of Lebesgue measure zero, J. Math. Anal. Appl. (in press).

[11] S. Czerwik, Stability of Functional Equations of Ulam-Hyers-Rassias Type, Hadronic Press, Inc., Palm Harbor, Florida, (2003).

[12] H. Drygas, Quasi-inner products and their applications, In: A. K. Gupta (ed.), Advances in Multivariate Statistical Analysis, 13-30, Reidel Publ. Co., (1987).

[13] B. R. Ebanks, PL. Kannappan and P. K. Sahoo, A common generalization of functional equations characterizing normed and quasi-innerproduct spaces, Canad. Math. Bull. 35, pp. 321-327, (1992).

[14] M. Fochi, An alternative functional equation on restricted domain, Aequationes Math. 70, pp. 201-212, (2005).

[15] G. L. Forti, J. Sikorska, Variations on the Drygas equations and its stability, Nonlinear Analysis, 74, pp. 343-350, (2011).

[16] R. Ger, J. Sikorska, On the Cauchy equation on spheres, Ann. Math. Sil., 11, pp. 89-99, (1997).

[17] S. -M. Jung, On the Hyers-Ulam stability of the functional equations that have the quadratic property, J. Math. Anal. Appl. 222, pp. 126137, (1998).

[18] S.-M. Jung, P. K. Sahoo, Stability of functional equation of Drygas, Aequationes Math. 64, pp. 263-273, (2002).

[19] S.-M. Jung, Hyers-Ulam-Rassias Stability of Functional Equations in Nonlinear Analysis, Springer, New York, (2011).

[20] M. Kuczma, Functional equations on restricted domains, Aequationes Math. 18, pp. 1-34, (1978).

[21] Y.-H. Lee, Hyers-Ulam-Rassias stability of a quadratic-additive type functional equation on a restricted domain, Int. Journal of Math. Analysis, Vol. 7, no. 55, pp. 2745-2752, (2013). 
[22] J. C. Oxtoby, Measure and Category, Springer, New York, (1980).

[23] J. M. Rassias, On the Ulam stability of mixed type mappings on restricted domains, J. Math. Anal. Appl. 281, pp. 747-762, (2002).

[24] J. M. Rassias, M. J. Rassias, On the Ulam stability of Jensen and Jensen type mappings on restricted domains, J. Math. Anal. Appl. 281, pp. 516-524, (2003).

[25] J. Sikorska, On two conditional Pexider functional equations and their stabilities, Nonlinear Anal. 70, pp. 2673-2684, (2009).

[26] J. Sikorska, On a direct method for proving the Hyers-Ulam stability of functional equations, J. Math. Anal. Appl. 372, pp. 99-109, (2010).

[27] D. Yang, Remarks on the stability of Drygas equation and the Pexiderquadratic equation, Aequationes Math. 68, pp. 108-116, (2004).

\author{
Muaadh Almahalebi \\ Department of Mathematics, \\ Faculty of Sciences, \\ Ibn Tofail University, \\ BP : 14000, Kenitra \\ Morocco \\ e-mail : muaadh1979@hotmail.fr
}

